\title{
El ciclo repetitivo en la Odontología Restauradora.
}

\author{
The repetitive cycle in Restorative Dentistry.
}

\author{
Carlos Carrillo Sánchez*
}

\section{RESUMEN}

Los cambios en la vida, así como también en el campo de la Odontología, deben ser para mejorar siempre en todos los aspectos posibles. Y muchos de los cambios recientes en la Odontología Restauradora están encaminados a la utilización de materiales estéticos para remplazo de restauraciones metálicas previas, sin generar certeza total de ser mejores opciones. Esto ha generado un marcado incremento en la tendencia a practicar, más enfáticamente, el remplazo de restauraciones previas.

Palabras clave: Cambios en la odontología, remplazo de restauraciones, causas de fracaso de restauraciones, caries secundaria.

Cuánto dura el para siempre - Le preguntó Alicia al conejo blanco.

A veces, sólo un segundo - Responde el conejo. Hay sueños que duran un instante en el que todo parece congelarse por una eternidad.

Lewis Carroll. Las aventuras de Alicia en el país de las maravillas.

\section{INTRODUCCIÓN}

$\mathrm{U}$ no de los problemas más grandes y preocupante que se puede encontrar en la Odontología Restauradora es la constante vivencia del ciclo repetitivo de las restauraciones dentales, el cual los dentistas generales han adoptado como parte inminente y fundamental de su práctica diaria y acentuándose más firmemente en los últimos años. ${ }^{1,2}$

El punto final al concluir el proceso de la colocación de restauraciones dentales normalmente es un paso que

\section{ABSTRACT}

Changes in life, as so in the field of Dentistry, should always be for the improvement on most possible aspects. Many of the recent changes in Restorative Dentistry are focus on the use of cosmetic materials to replace previous metallic restorations, without total certainty of being better options. This has generated a marked increase in the tendency to practice, more emphatically, the replacement of previous restorations.

Keywords: Changes in dentistry, replacement of restorations, causes of failure of restorations, secondary caries.

\footnotetext{
* Maestría en Ciencias Dentales. Práctica privada. Toluca, Estado de México. México.

Recibido: 25 de agosto de 2020. Aceptado: 16 de agosto de 2021.

Citar como: Carrillo SC. El ciclo repetitivo en la Odontología Restauradora. Rev ADM. 2021; 78 (5): 283-290. https://dx.doi.org/10.35366/102037
}

llena de mucha satisfacción al dentista y, además, éste lo hace percibiendo la idea de ser algo que va a formar parte de la integridad total, íntima y permanente del diente restaurado.

Por desgracia, es imperante tener que repasar de manera más firme este concepto y asimilar que las restauraciones tienen un lapso o periodo de vida muy limitado, dependiendo mucho de factores diversos, fundamentales y muy importantes, como son: las variables en el material restaurador, en el mismo operador y del paciente en sí, que pueden fácilmente traducirse en el aspecto clínico en fracasos al aflorar imperfecciones propias de los materiales dentales utilizados, deficiencias técnicas en la restauración o la recurrencia de caries. Los dentistas lo deben tener en mente, mucho antes de ofrecer una nueva restauración..$^{2,3}$

Las nuevas expectativas de vida de las generaciones actuales requieren de tener que buscar recursos más exhaustivos y científicos que prevengan o limiten lo más posible este ciclo de vida tan repetitivo que sólo ha gene- 
rado la presencia de bocas altamente restauradas, afectadas y mutiladas propias de las generaciones previas. $^{3-5}$

\section{ANTECEDENTES}

Es muy válido pensar que la Odontología Restauradora es muy necesaria y esta idea debe prevalecer, aunque a esta postura se contrapongan varios aspectos. Porque también es muy válido entender que la Odontología Restauradora sufre y presenta por sí misma de un buen número de características de fracasos; pero para entender esto, es necesario tener siempre muy en mente que sin restauraciones, muchos dientes que fueron «salvados» habrían presentado problemas más serios o hubieran sido condenados a perderse prematuramente. ${ }^{4,5}$

La Odontología Restauradora ha prevalecido con mucho éxito por más de 200 años. Y su desarrollo fue producto de la necesidad imperante de tratar de controlar la enfermedad o pandemia de la caries y del entendimiento del poco beneficio que se generaba a la población con la odontología mutilante, en la llamada «Era de las Extracciones».

Desde su nacimiento, el núcleo fundamental de la educación dental en la mayoría de las instituciones en el mundo se ha enfocado en la Odontología restauradora. ${ }^{4}$

$Y$ aun cuando es mal comprendido o poco difundido, gracias al entendimiento científico y la aplicación de los principios del Dr. GV Black la Odontología Restauradora ha prevalecido, pudiendo convertirse con el paso de los años en una Odontología más conservadora y más exitosa. Pero, no se ha conseguido enfocar mucho o bien dirigidas las bases de estos principios para lograr conquistar finalmente la práctica de una Odontología más preservadora.

Sigue existiendo y prevaleciendo, a pesar de ser un concepto totalmente incorrecto, la idea de que sólo las preparaciones muy extensas, con ángulos internos agudos y con una extensión por prevención amplia son las que regulan los principios desarrollados por el Dr. Black para poder retener por sí mismas y en buena función a un material restaurador directo. Esto ha generado que los dentistas piensen que los problemas principales de los fracasos de las restauraciones sean cuestiones mecánicas y que, por lo tanto, se tengan que resolver o evitar por medio de métodos técnicos similares.

De ahí que se vea desde hace ya muchos años a las restauraciones dentales como «panacea» y se considere el resultado propio y como única solución para obtener la deseada salud dental, y que la enfermedad de la caries se «cura» con la colocación de restauraciones. Para muchos, la salud dental es sinónimo de boca con restauraciones.
Además, sigue prevaleciendo el adagio de: «cortar o preparar y llenar o restaurar» como única solución para sanar la enfermedad de la caries.

Es necesario entender que la evolución propia de la Odontología, y en especial de la Odontología Restauradora, se debe a cambios en muchos aspectos, los cuales deben ser aceptados como parte fundamental de su evolución normal y propios de los avances científicos de la profesión dental. Estos cambios son:

1. Niveles de caries.

2. Entendimiento de la ciencia de la caries.

3. Apreciación de las deficiencias o errores de la odontología restauradora tradicional.

4. Materiales dentales y en las técnicas restauradoras.

5. Prácticas dentales por el surgimiento de nuevas enfermedades.

6. Requerimientos para un apropiado control de infección cruzada.

7. Aspiraciones o expectativas de los pacientes y que deben valorarse con base en la cosmética y a favor de la misma o de la salud oral.

Se tiene que entender también que si se ha logrado reducir la incidencia de caries, la posibilidad de interceptar un proceso de caries en forma más temprana, y si ha podido existir y prevalecer la posibilidad de enseñar y desarrollar una Odontología Restauradora más conservadora, es gracias a la aceptación de medidas preventivas que forman, desde hace algunos años, parte fundamental de la vida diaria de buena parte de la población, como: con fluoruros en sus distintas opciones de uso o de acceso, control de placa bacteriana o biofilm microbiano y una mejor educación nutricional. Pero tampoco esto ha logrado generar una conciencia más plena en la profesión dental para evitar que el ciclo repetitivo de las restauraciones deje de ser una parte fundamental de la práctica de la Odontología Restauradora. ${ }^{2,4}$

\section{CONCEPTOS GENERALES}

En la práctica dental diaria, se puede constatar que la mayor parte del tiempo de trabajo de los dentistas generales está dedicado al remplazo de restauraciones, generado por múltiples y diversas razones muy comunes, pero muchas veces sin fundamentos adecuados. ${ }^{3,5}$

La mayoría de las restauraciones duran mucho menos tiempo de vida en boca de las expectativas generales esperadas, y normalmente el tiempo promedio estimado, en el mejor de los casos, es de aproximadamente siete 
años. Un tiempo muy corto y muy limitado para la función deseada. Además, se manejan datos de que, de tres restauraciones colocadas en la práctica general, una es considerada insatisfactoria o al menos que no cumple con todos los requerimientos para considerar que pueda llegar a completar esos siete años sin presentar algún problema que requiera su remplazo previo. ${ }^{2,4}$

Los materiales dentales restauradores son por naturaleza imperfectos o limitados en sus propiedades ideales, y si a eso se le aúnan las posibles fallas técnicas o el uso
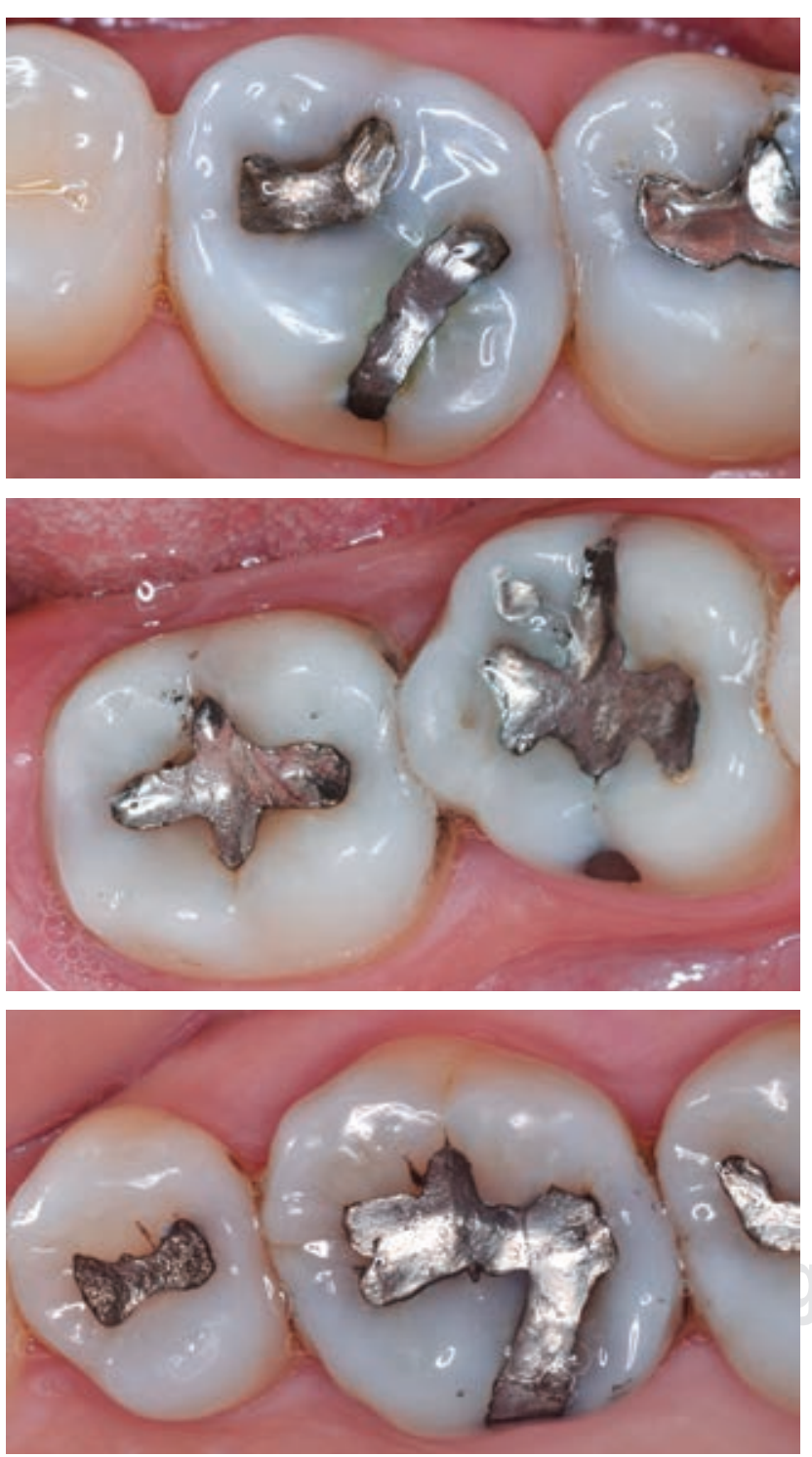

Figura 1: Restauraciones de amalgama en paciente de 35 años, que pueden ser mantenidas así por muchos años sin requerir ser remplazadas. poco óptimo durante su colocación, muchas restauraciones están condenadas a presentar fracasos previos, aun desde recién colocadas. ${ }^{3,5}$

Elderton $^{4}$ menciona los siguientes factores como preponderantes a causar los fracasos y la importancia de ponerlos en consideración siempre antes de efectuar una restauración dental o de efectuar algún remplazo:

1. Los materiales dentales son, por sí mismos, imperfectos.

2. La destreza clínica y la motivación necesaria para su uso óptimo son insuficientes.

3. La medición objetiva de las restauraciones demuestra que una gran cantidad de ellas son mediocres o deficientes.

4. Muchos dentistas buscan, en forma rápida, remplazar restauraciones que se juzgan imperfectas.

5. Se busca remplazar restauraciones a pesar de que no se pueda establecer la causa real del problema, o aun cuando éste no exista.

6. Es muy común repetir el problema original del «fracaso».

7. Mientras que en el proceso, el tamaño de la cavidad aumenta considerablemente y se genera mayor daño.

Existe una tendencia muy clara a emitir juicios poco sólidos para la valoración clínica de restauraciones presentes en boca. Por lo general, el dentista clínico tiende a evaluar más las restauraciones previas en cuanto a cuáles deben ser removidas, por sobre cuáles deben ser conservadas, mantenidas o mejoradas, aun cuando no se logre tener un concepto muy claro de la posible causa que marcaría el fracaso. $2,3,5$

El Dr. Eduardo Ortega Zárate (QEPD), entrañable maestro y gran experto en la ciencia de los materiales dentales, solía comentar que las restauraciones de amalgama dental son feas por fuera, pero muy bonitas por dentro. $\mathrm{Y}$ esto es gracias a la capacidad que presenta la amalgama dental de generar productos de corrosión que sellan la interfase entre el material restaurador y la estructura dental. ${ }^{i}$ Algo que, aún en nuestros días, ningún material dental restaurador ha podido desarrollar. La adhesión que se logra con los materiales dentales restauradores actuales tiende a disminuir bajo las condiciones adversas presentes y propias de la cavidad oral.

En estudios longitudinales se ha encontrado que $46 \%$ de las restauraciones colocadas en los consultorios dentales se deben a caries primaria, mientras que el resto (54\%) son remplazos de restauraciones con diagnóstico de

${ }^{i}$ Ortega Zárate E. Comunicación personal. 1976. 


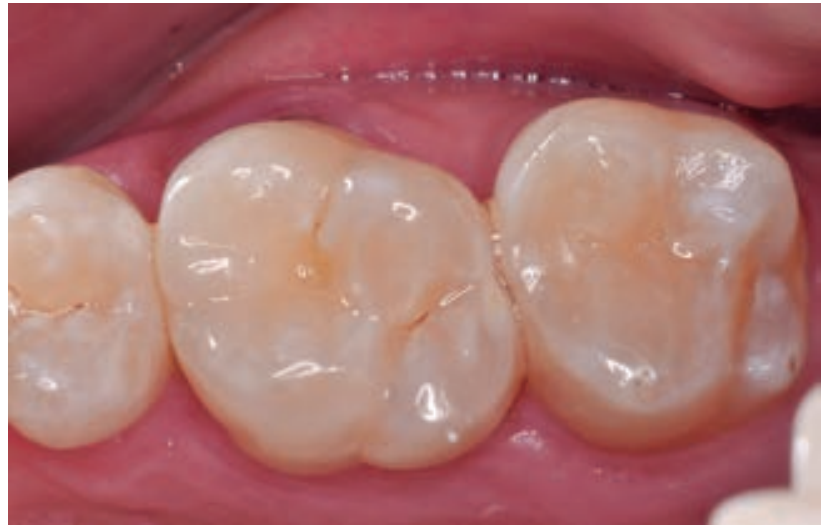

Figura 2: Paciente de 35 años que ha mantenido sus dientes sin caries y que pueden tener mejor expectativa de vida sin restauraciones.

fracasos no muy claros, ${ }^{2}$ siendo este número demasiado alto para las expectativas forjadas.

Las principales razones que se llegan a considerar más comúnmente como causales de fracasos en restauraciones son:

1. Una carga oclusal excesiva.

2. Mal diseño de la preparación o errores en su diseño previo.

3. Pobre manejo de los tejidos, tanto duros como blandos.

4. Una pobre selección de los materiales dentales utilizados,

5. Y obviamente, se debe considerar la importancia de la presencia de caries secundaria si han prevalecido las condiciones propias para el desarrollo de la enfermedad. $2,4,6$

Sólo se pueden excluir estas razones cuando es obvio un fracaso catastrófico o la presencia persistente de dolor o molestia constante. ${ }^{7,8}$

La presencia de caries secundaria ha sido reportada como la más importante de las razones para el remplazo de restauraciones dentales, aun sin considerar la precisión con la que se efectúe el diagnóstico. Ha sido muy recomendado mejorar e implementar la mayor cantidad posible de métodos de detección de lesiones de caries para ayudar a elaborar un diagnóstico más preciso de la presencia de caries secundaria, siempre sin mucho éxito (Figura 1). ${ }^{1-3,8}$

Los dentistas generales no hacen un buen esfuerzo para aplicar de mejor forma los métodos de diagnóstico que puedan ayudar a tener muy claro si una restauración debe ser condenada al remplazo, principalmente para hacer una detección más precisa sobre la presencia de caries recurrente o secundaria. Los pacientes están pagando un precio muy alto por malos diagnósticos o tratamientos imprecisos, sobre todo cuando suelen cambiar con frecuencia de dentistas y no se llega a considerar el manejo especial que el dentista previo pudiera estar llevando sobre el plan de tratamiento en particular (Figura 2), ${ }^{4,6,8}$ deben ser orientados a entender este problema, en particular cuando el odontólogo ha implementado cierto manejo especial sobre alguno o varios dientes en el tratamiento, si el odontólogo ha tomado una conciencia muy particular al observar la evolución de algunas restauraciones que puedan presentar cierto deterioro; y es porque tiene familiaridad sobre el caso o puede estar considerando el pronóstico y la posible evolución que pueda presentar ese diente y su valor al conservarlo. Mientras que un odontólogo que ve por primera vez a ese paciente puede no considerar tomar riesgos adversos que podrían haber resultado en un mayor beneficio para el paciente (Figura 3).9-11

Esto es algo que se ve con mucha frecuencia, el odontólogo que recibe a un paciente nuevo y lo ve por primera vez fundamenta su plan de tratamiento sin considerar la posibilidad de efectuar un manejo a largo plazo de los dientes que presentan restauraciones previas, sino que las considera únicamente sobre la posibilidad de su necesidad de remplazo. ${ }^{3,8,9}$

También los estudios longitudinales sobre el remplazo de las restauraciones han demostrado que el remplazo de restauraciones de amalgama dental por la presencia de caries secundaria es de entre 29 y 53\%, mientras que

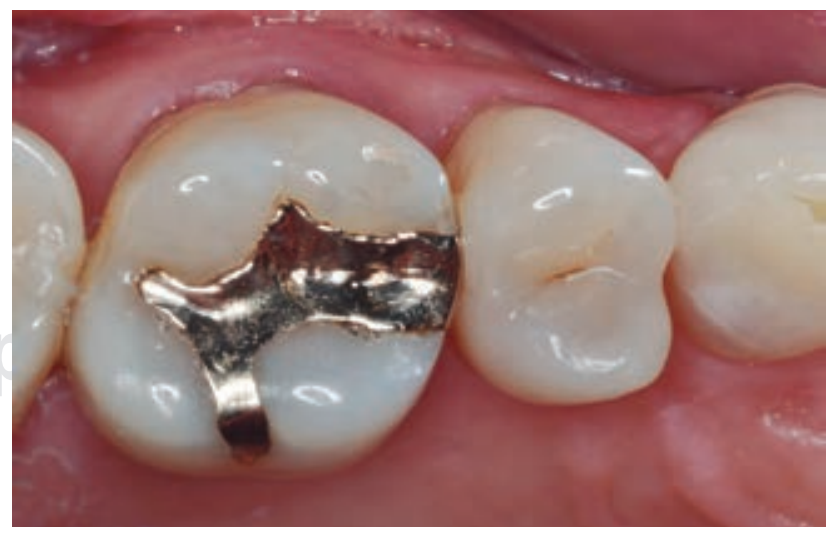

Figura 3: Paciente adulto con restauración de incrustación metálica con más de 45 años en boca y funcionando sin ningún problema. 


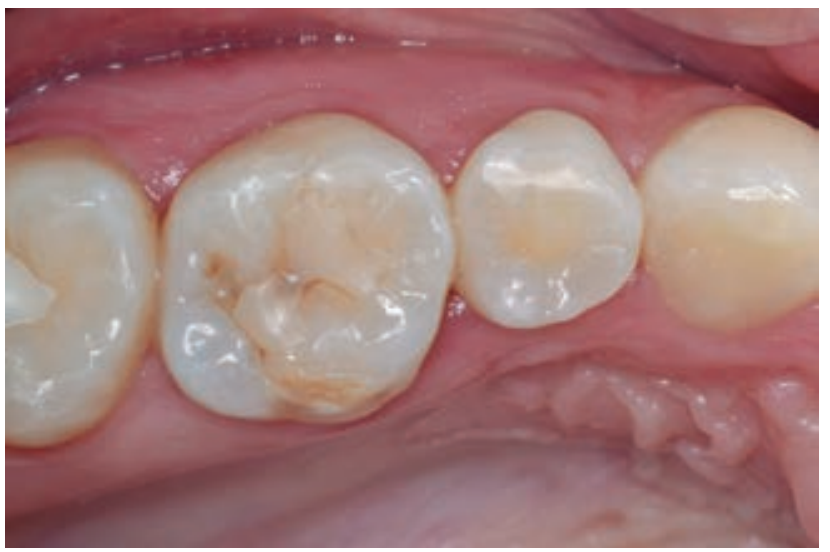

Figura 4: Restauración de resina compuesta no muy exitosa que remplazó a una amalgama dental y con pobre pronóstico de longevidad.

para las restauraciones de resinas compuestas es de 40 y $45 \%$. Y se sigue sentenciando más a las amalgamas dentales buscando su remoción por el hecho principal de ser poco estéticas y, sobre todo, por la presencia de caries secundaria (Figura 4).2,5,10

A pesar de ser un número alto, se puede pensar que en las restauraciones de resinas compuestas, la presencia de lesiones de caries secundaria no es una de las razones principales que se consideran para sugerir su remplazo, porque algunos otros factores son preponderantes y se consideran los fracasos debido a deficiencias en la técnica utilizada en la colocación o por las fallas en las propiedades físicas y químicas del material per se. .,10,11 $^{-11}$

Lo importante a considerar en estos enunciados previos es que, dentro de los fracasos de las restauraciones condenadas a ser remplazadas, es necesario hacer una mejor evaluación sobre las causas principales de fracaso y considerar mejor el servicio que están proporcionando esas restauraciones y tomar mayor conciencia de evitar crear restauraciones que tengan que ser remplazadas en corto tiempo.

\section{CONCEPTOS ACTUALES}

El diagnóstico de caries secundaria debe girar con base en nuevos paradigmas, y lo mismo debe ser aplicado a las lesiones de caries incipientes. ${ }^{1,2}$ No retirar una restauración previa por presencia de caries secundaria hasta no tener la certeza de la presencia de caries en dentina y de que sea caries; así como en el caso de una lesión de caries primaria, no abrir una preparación en ese diente hasta tener la plena certeza de la presencia de una lesión de caries activa. En el entendido de que es más difícil la detección de una lesión de caries secundaria que la presente en un diente virgen y en el que, al remover una restauración previa, tenemos que remover mayor estructura dental adicional (Figura 5). ${ }^{11,12}$

La idea fundamental que debe prevalecer en toda práctica dental actual debe ser la de buscar proporcionarle a todos los pacientes de cualquier edad la oportunidad de mantener una dentición saludable y funcional de por vida.

Esta idea debe estar basada y prevalecer, bajo los conceptos de: «prevenir lo que es prevenible y detener las enfermedades o daños presentes bajo el sustento de utilizar los recursos de tratamientos más eficientes y con bases sólidas de buena evidencia científica».5, 6

Elderton ${ }^{4}$ también considera importante tener muy en claro los siguientes postulados al efectuar Odontología Restauradora:

1. «La decisión de emprender tratamiento restaurador sólo puede ser apropiada si al mismo tiempo se aplican medidas preventivas».

2. «Cuando sea necesario emprender el tratamiento restaurador, debe ser considerado siempre como una medida preventiva primaria».

Bien se dice que «la fresa dental puede remover en pocos segundos más estructura dental, que lo que la caries puede alterar o dañar en meses o años».

Se debe tener también muy claro que no se puede ser muy conservador al momento de entrar en el ciclo repetitivo de restauraciones dentales, además de considerar que con cada remplazo de una restauración, se

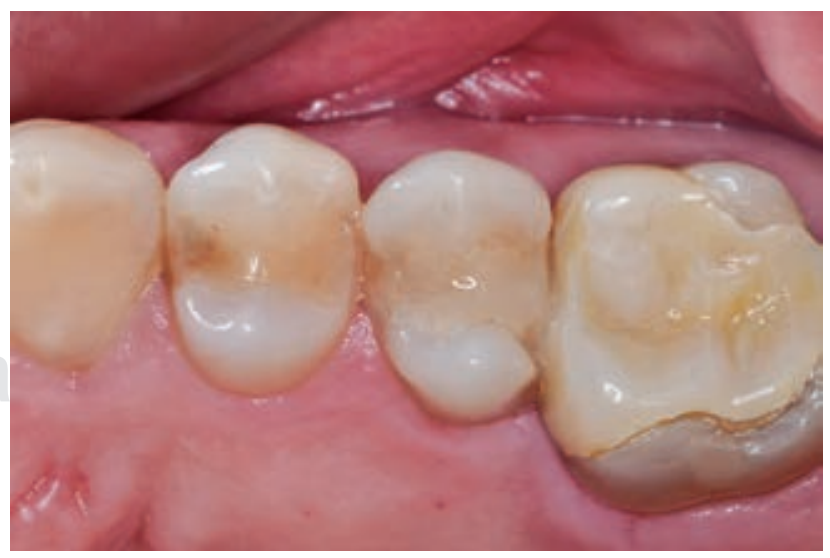

Figura 5: Remplazos con materiales estéticos que no demuestran tener buen pronóstico a corto plazo. 
destruye más estructura dental, se remueven cúspides, el diente se vuelve más frágil, el procedimiento es más complicado, se vuelve más costoso, se puede involucrar a la pulpa dental, probablemente se requerirá de otro tipo de procedimientos más largos, difíciles e invasivos y se incrementa la posibilidad de nuevos fracasos. $5,13,14$

Es muy necesario recordar que la restauración de la cavidad no es una «cura» para la enfermedad de la caries, porque prevaleciendo las condiciones, puede reaparecer una o más veces alrededor de la restauración, ya sea como una nueva lesión o como caries residual cuando no fue completamente removida la lesión previa durante la restauración del diente, o ese mismo diente puede desarrollar nuevas lesiones de caries en estructura dental intacta. ${ }^{2,4,6}$

Para obtener la máxima longevidad de las restauraciones, el odontólogo debe tener muy clara la técnica a implementar, los procedimientos operatorios bien probados y aceptados científicamente, poner en práctica sus mejores habilidades, utilizar los mejores materiales dentales con base en evidencia científica e implementar y motivar a los pacientes a seguir un programa de odontología preventiva que debe ser especialmente diseñado para cada uno de ellos.

En algunos países de la Comunidad Europea y en el Reino Unido, se considera de manera muy abierta buscar preservar las restauraciones el mayor tiempo posible sin intentar su remoción total o sacrificar su función, con base en recontorneos, remarginaciones, mejoramientos y reparaciones; y aun cuando el implementar estas técnicas ha generado controversias fuertes en las instituciones de enseñanza y sociedades dentales, existen reportes de significancia de éxito y de gran aceptación por parte de odontólogos en estos países.9,14 En las escuelas de odontología y en las asociaciones profesionales americanas, así como en los países donde las bases de su enseñanza dental se fundamentan en lo prevalente en los Estados Unidos de Norteamérica, el manejo de las restauraciones bajo esta premisa ha sido considerada poco profesional o científica, pero en los últimos años se ha podido llegar a considerar como una muy buena alternativa previa a efectuar el remplazo total de las restauraciones (Figura 6). ${ }^{15}$

Simon y George ${ }^{16}$ toman muy en cuenta y recomiendan el tratar de implementar lo más posible durante la colocación de resinas compuestas en dientes posteriores el seguimiento de los pasos clínicos con mucha precisión en su manejo, evitando caer en errores durante el procedimiento que puedan ser perjudiciales, y consideran fundamental: conocer perfectamente las instrucciones sobre el material restaurador y el material adhesivo re-

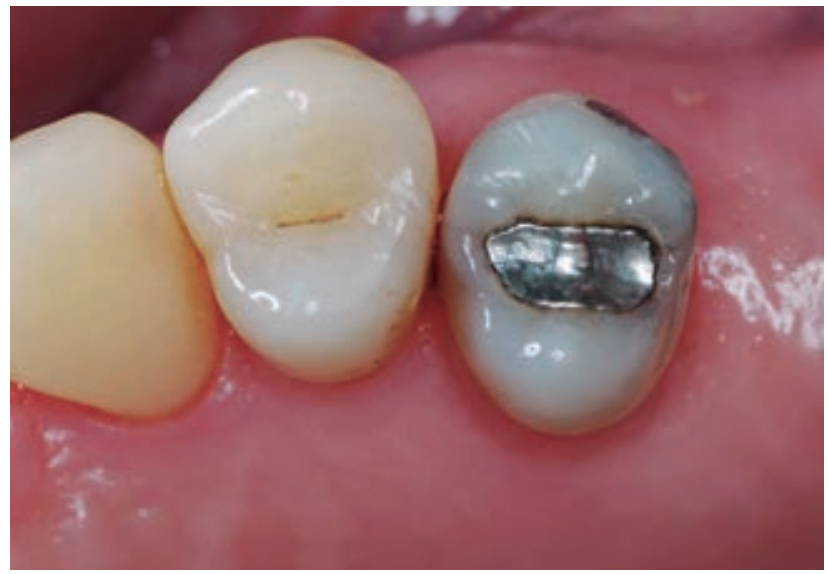

Figura 6: Restauración de amalgama dental con un istmo amplio, pero con más de 70 años en boca.

comendado, así como los diferentes pasos en su manejo, tener un efectivo control del campo operatorio, controlar y evitar generar contracción a la polimerización, seguir un adecuado proceso del curado del material por luz, controlar en buena medida y cuando sea posible el tamaño y la ubicación de la restauración, utilizar una técnica de colocación precisa por incrementos, desarrollando un adecuado manejo de las cajas proximales (con la posibilidad de dividir la restauración en diversas porciones, proximales, axiales y sólo al final la porción oclusal) y posponer el pulido y terminado de la restauración cuando sea posible. Esto es, tener un mayor control sobre las variables que puedan interferir en el éxito de la restauración y evitar problemas muy comunes inicialmente, como microfracturas del esmalte o sensibilidad postoperatoria.

Brady ${ }^{17}$ recomienda tener muy en consideración las posibilidades de fracaso e intentar ir evitando esas posibilidades para no generar cercanías a fracasar. Ella sugiere definir de manera clara lo que significa para cada uno el fracaso y enfocar los tratamientos en la valoración del resultado obtenido en contraposición con las expectativas fijadas. De ese modo, Brady maneja el evitar crear restauraciones que, dentro de sus expectativas a corto o largo plazo, puedan considerarse como fracasos. Y esto ayuda a que los pacientes puedan tener, desde el principio del tratamiento, el conocimiento de cuánto tiempo pueden durar sus restauraciones, y en buena parte sirve para hacerlos participes y corresponsables del éxito de la restauración (Figura 7).

Arvanitis ${ }^{18}$ considera que gran parte de los errores en el diagnóstico de problemas que puedan presentar los dientes con restauraciones recaen en que los dentistas 
tienden a utilizar los métodos de diagnóstico convencionales y que se han utilizado por muchos años y a través de muchas generaciones sin perfeccionar, pero que son recomendados y han sido sugeridos para la detección de lesiones primarias en dientes sin restauración. Además, es muy necesario tratar de adecuar y estandarizar lo más posible los criterios propios del diagnóstico y acercar a que las diferencias o variables entre los mismos dentistas sean más pequeñas y menos significativas. ${ }^{6}$

Se vuelve básico en este ciclo repetitivo de las restauraciones dentales el entender que la longevidad de las restauraciones debe estar cimentada en la consideración de los riesgos técnicos del procedimiento, los riesgos propios que presentan el paciente, el diente y la misma restauración, y valorarlos dentro de una curva de campana que representaría la distribución de valores, frecuencias y probabilidades a considerar como lo que sería la esperanza de expectativa de vida. Y al final, generar sólo expectativas confiables y realistas.

\section{CONCLUSIONES}

Algo muy importante a considerar en nuestros días en favor de la Odontología Restauradora es que la prevalencia de la enfermedad de caries ha cambiado dramáticamente a partir de los años 50 del siglo pasado, lo que nos permite no tomar decisiones equivocadas o tan drásticas, y que la incidencia de caries ha tenido una tendencia natural a disminuir y que los materiales dentales restauradores han mejorado ampliamente en su calidad, además de que los tratamientos dentales restauradores muy invasivos pueden ser evitados y se deben declinar o posponer a favor de ser

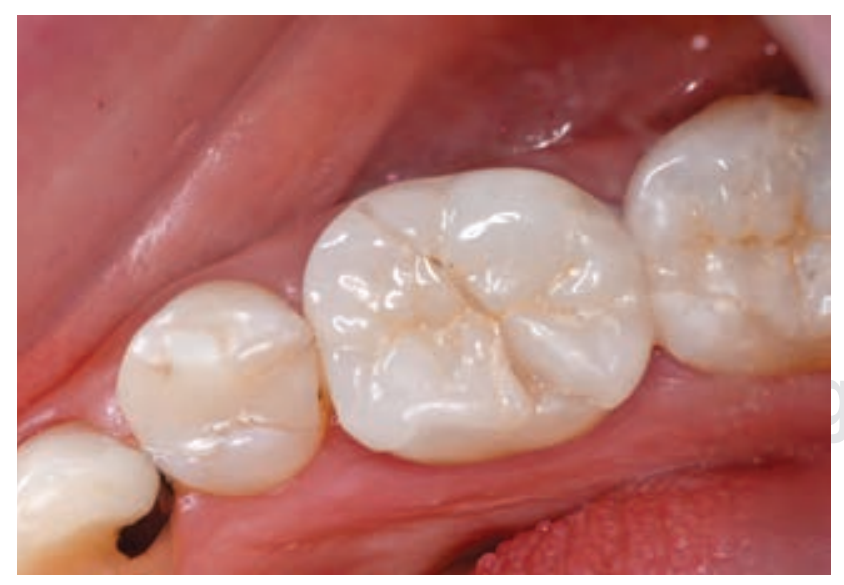

Figura 7: Restauración con incrustación cerámica no podría ser la mejor selección para la función masticatoria en este paciente. substituidos por medidas o tratamientos más preventivos. Esto puede ayudar y mejorar considerablemente para generar y tener expectativas de vida más largas de los órganos dentarios en la boca de los pacientes.

Como medida interna primordial, una práctica profesional enfocada a la Odontología Restauradora debe adoptar sistemáticamente la estandarización de mejorar la calidad de las restauraciones en forma congruente y compaginada con la medición del índice de riesgo de caries y basada en el adecuado manejo de caries de cada paciente en particular; ${ }^{1,3,19}$ mientras que la investigación odontológica trabaja en el desarrollo de nuevos instrumentos, métodos y procedimientos que ayuden a crear certidumbre y precisión de la funcionalidad o deterioro de las restauraciones existentes en boca.

El poder determinar un régimen de tratamiento basado en el manejo del riesgo de caries proporcionará una mayor probabilidad de éxito, mayor tiempo de vida de las restauraciones, así como generar un mejor costoefecto, aun antes de pensar en aplicar el concepto de «Odontología de invasión mínima». ${ }^{11}$

Por lo tanto, es importante considerar que deben prevalecer como viables y factibles las recomendaciones de Gunnar Ryge de hace más de 50 años, sobre evaluar las restauraciones basándose en las características y propiedades innatas de los materiales dentales restauradores y su relación con la estructura dental soportante, como son:

1. Similitud en igualdad de color del diente.

2. La decoloración presente en los márgenes.

3. La forma anatómica prevaleciente.

4. La adaptación marginal.

5. La detección de caries, principalmente en la zona marginal. ${ }^{13}$

Siempre y cuando se pueda sustentar que el diente y su restauración van a mantener sus principios y propiedades funcionales, biológicas y estéticas. Y considerar también la importancia de poder tratar de contar con una guía más específica que ayude a la categorización de las restauraciones previas en su calidad o servicio y no sólo con base en un comportamiento satisfactorio o una estética inaceptable, como:

1. En la probabilidad y significancia clínica de deterioro o de desarrollo de una lesión.

2. La contraindicación clara de intentar su mejoramiento o reparación.

3. Que el beneficio del remplazo supere por mucho el posible efecto o daño por permanecer. 
4. Que existan los pronósticos favorables en el comportamiento clínico.

5. El entendimiento y la aceptación por parte del paciente.

Por lo tanto, buscar desechar también el viejo adagio de: «en la duda, remover y remplazar»; substituirlo y adoptar el más moderno de: «sólo como un último recurso, remplazar».

Pero en pacientes de primera vez con restauraciones previas y sin una idea muy clara de causas de deterioro en las restauraciones, se hace muy viable la opción de sustentar mejor la medición de riesgo de caries con la posibilidad de incorporar en las restauraciones con duda con base en «observar y valorar». ${ }^{9,19}$ Esta misma filosofía debe ser incorporada a una visión más moderna que deberá prevalecer en el futuro y que debe ser:

1. Menos odontología puramente mecánica.

2. Adoptar mayores y mejores bases científicas.

3. Demostrar una mayor sensibilidad humana.

4. Desarrollar la satisfacción de ser odontólogos más conservadores y preventivos.

La práctica de la Odontología Restauradora moderna debe encausar por sí misma al odontólogo a desarrollar una visión más preservadora, utilizando mejores medidas y técnicas de diagnóstico y evitar así condenar fácilmente a las restauraciones previas a tener que ser remplazadas.

Tomar en consideración los principios que fomenten los nuevos conceptos restauradores más actuales que guíen la Odontología al llamado «concepto dinámico restaurador» en la búsqueda primordial de la longevidad de los dientes por sobre la longevidad de las restauraciones dentales. ${ }^{15}$

Markley ${ }^{\mathrm{ii}}$ solía comentar que las restauraciones dentales deben ser tanto una reparación como un tratamiento de la caries y que las buenas restauraciones deben limitar la enfermedad y al mismo tiempo reparar el daño, y que ese tipo de restauraciones debe permanecer por muchos años y ayudar a preservar la salud y evitar la enfermedad.

\section{REFERENCIAS}

1. Carrillo SC. Diagnóstico de lesiones incipientes de caries ¿Es este el futuro de la Odontología? Rev ADM. 2010; 67 (1): 13-20.
2. Carrillo SC. La caries secundaria y su adecuado diagnóstico. Rev ADM. 2012; 69 (6): 258-265.

3. Mjor IA, Toffenetti F. Secondary caries: a literature review with case reports. Quintessence Int. 2000; 31 (3): 165-179.

4. Elderton R. Current understanding of oral diseases and abnormalities. In: Evolution in dental care. Chapter 3. Bristol, UK: Clinical Press Limited; 1990. pp. 13-36.

5. Wilson NA, Whitehead SA, Mjor IA, Wilson NH. Reasons for the placement and replacement of crowns in general dental practice. Prim Dent Care. 2003; 10 (2): 53-59.

6. Elderton R. Principles of decision-making to achieve oral health. In: Anderson M. Professional prevention in dentistry. Advances in Dentistry 1. Baltimore, MD: Williams and Wilkins; 1994. pp. 3-27.

7. Lutz F. Alternatives to amalgam in posterior teeth. In: Duke ES, editor. The changing practice of restorative dentistry. Proceedings of the 5th. Indianapolis, IN: Annual Indiana Conference. Indiana University School of Dentistry; 2002. pp. 263-274.

8. Devlin H. Operative dentistry. A practical guide to recent innovations. Chapter 2. Berlin: Springer; 2006. pp. 17-47.

9. Mjor IA. Reasons for restoration failures. In: Duke ES, editor. The changing practice of restorative dentistry. Proceedings of the 5th. Indianapolis, IN: Annual Indiana Conference. Indiana University School of Dentistry; 2002. pp. 47-55.

10. Wilson $\mathrm{NH}$, Brunton PA. Reducing the incidence of secondary caries. In: Duke ES, editor. The changing practice of restorative dentistry. Proceedings of the 5th. Indianapolis, IN: Annual Indiana Conference. Indiana University School of Dentistry; 2002. pp. 255-262.

11. McComb D. Conservative operative management strategies. Dent Clin North Am. 2005; 49 (4): 847-865, vii-viii.

12. Bayne S. Clinical decisions using the scientific method. In: Duke ES, editor. The changing practice of restorative dentistry. Proceedings of the 5th. Indianapolis, IN: Annual Indiana Conference. Indiana University School of Dentistry; 2002. pp. 13-26.

13. Ryge R, Cvar J. Criteria for the clinical evaluation of dental restorative materials. U.S. Public Service 790244. San Francisco, CA: Government Publishing Office; 1970. pp. 1-42.

14. Wilson N, Lynch CD, Brunton PA, Hickel R, Meyer-Lueckel H, Gurgan $\mathrm{S}$ et al. Criteria for the replacement of restorations: Academy of Operative Dentistry European Section. Oper Dent. 2016; 41 (S7): S48-S57.

15. Opdam N, Hickel R. Operative dentistry in a changing dental health care environment. Oper Dent. 2016; 41 (S7): S3-S6.

16. Simon JF, George LA. Factors for successful composite restorations. Inside Dentistry. 2014; 10 (6): 1-6.

17. Brady LA. Avoiding restorative failure. Dent Today. 2013; 32 (3): 88-91.

18. Arvanitis G. Criteria for the replacement of defective restorations. Dent Today. 2004; 23 (4): 78-81.

19. Mjor IA, Wilson NHF. Carious lesions: management alternatives. J Oper Dent. 2001; (Suppl 6): 237-241.

Correspondencia:

Dr. Carlos Carrillo Sánchez, MSD

E-mail: drcarrillocdmsd@gmail.com

ii Markley M. Comunicación personal. 1983. 